\title{
Onset of thermal ripples at the interface of an evaporating liquid under a flow of inert gas
}

\author{
B. Scheid ${ }^{1}$, J. Margerit ${ }^{2}$, C.S. Iorio $^{3}$, L. Joannes ${ }^{4}$, M. \\ Heraud $^{4}$, P. Queeckers ${ }^{3}$, P.C. Dauby ${ }^{2} \&$ P. Colinet ${ }^{1}$ \\ 1 TIPs-Fluid Physics, Université Libre de Bruxelles C.P. 165/67, \\ Avenue F.D. Roosevelt, 50 - 1050 Bruxelles \\ 2 Institut de Physique B5a, Université de Liège, \\ Allée du 6 Août 17, B-4000 Liège, Belgium \\ 3 Microgravity Research Center, Université Libre de Bruxelles C.P. 165/62, \\ Avenue F.D. Roosevelt, 50 - 1050 Bruxelles \\ ${ }^{4}$ Lambda-X, Zoning II, Rue de l'Industrie 37, B-1400 Nivelles, Belgium
}

(Dated: November 10, 2011)

\begin{abstract}
The dynamics of thermal ripples at the interface of a volatile pure liquid $\left(\mathrm{C}_{2} \mathrm{H}_{5} \mathrm{OH}\right)$ is studied experimentally and numerically. Liquid evaporates under a flow of inert gas $\left(\mathrm{N}_{2}\right)$ circulating along the interface. The evaporation rate is varied by regulating both the gas flow rate and the gas pressure. Experiments in microgravity environment allowed to identify a transition to "interfacial turbulence", along which some particular events such as nearly periodic and possible intermittent behaviors. Direct numerical simulations have been performed, and compare qualitatively well with experimental results, offering new insights into the physical mechanisms involved. Small-scale ripples appear to arise from a secondary instability of large-scale convection cells and their motion seems to follow the corresponding large-scale surface flow. The relative role of surface tension and buoyancy in triggering these flows is assessed by comparing experiments and simulations in both microgravity and ground conditions. Qualitative features compares satisfactorily well such as typical speed and orientation of the thermal ripples, as well as spiral flow in the bulk.
\end{abstract}

Keywords: Marangoni effect, Interfacial turbulence, Evaporation, Microgravity, Pattern formation 


\section{INTRODUCTION}

Evaporation plays a central role in various industrial applications (see e.g. [1] or [2]), such as solvent evaporation in coating, paints or spray drying, falling film evaporators, etc. Most often, the liquid evaporates into air, or more generally an inert gas, which implies a limitation of the evaporation rate by diffusion of vapor into the inert gas $[3,4]$. However, as studied in Haut and Colinet [3], the presence of an inert gas also strongly favors surfacetension-driven (thermal Marangoni) instabilities in the liquid, which can enhance the heat transfer through the liquid phase, hence the evaporation rate.

Based on classical papers on evaporative convection driven by Marangoni effect (see e.g. [5-7]), destabilizing effects such as surface deformability, vapor recoil and departure of the interface from chemical potential equilibrium are all negligible when, respectively, the liquid layer is not too thin (some millimeters), the evaporation rate is not too large, and the kinetic resistance to evaporation can be neglected against heat and mass transfer resistances [3]. Those conditions constitute the framework in which experiments and simulations are conducted and described in the present paper. The main goal is to investigate thermal structures at the liquid/gas interface during the evaporation process, and especially at evaporation rates corresponding to high Marangoni numbers - the low Marangoni regimes having already been extensively studied (see [8, 9], and references therein).

Interfacial thermal structures in evaporating ethyl alcohol were first observed in microgravity conditions during the ITEL-Maser 9 sounding rocket experiment $[10,11]$. However, the evaporation rate could not be controlled and was fixed at a high value - i.e. low gas pressure and large gas flow rate - such that only chaotic behaviors were visualized, during a short time. The ITEL-2-Maser 10 experiment [12] allowed us to scan the evaporation rate from low to moderate values and therefore to scrutinize the transition toward chaotic behavior, along with various interesting features of the flow presented in this work. Even though the results obtained experimentally are scarce and transient in nature (due to the limited microgravity time available), it is possible to reach some important conclusions, especially by comparing them qualitatively with numerical simulations.

Besides the characterization of the interfacial thermal structures, another question that we attempt to answer qualitatively in the present work is whether thermal patterns efficiently mix the liquid and are influenced by buoyancy effects. 
The paper is organized as follows: the problem configuration is described in §II, experimental observations are detailed in $\S I I I$, experimental data are analyzed in $\S I V$, results of numerical simulations are given in $\S \mathrm{V}$, and concluding remarks are outlined in $\S \mathrm{VI}$.

\section{EXPERIMENTAL PRINCIPLE AND METHODS}

The experimental set-up has been presented in great details, including many schematics, by Janson et al.[12]. Here, we only present in Fig. 1 the geometry of the physical problem considered in the present paper. Ethyl alcohol $\left(\mathrm{C}_{2} \mathrm{H}_{5} \mathrm{OH}\right)$ is evaporated through a $15 \mathrm{~mm}-$

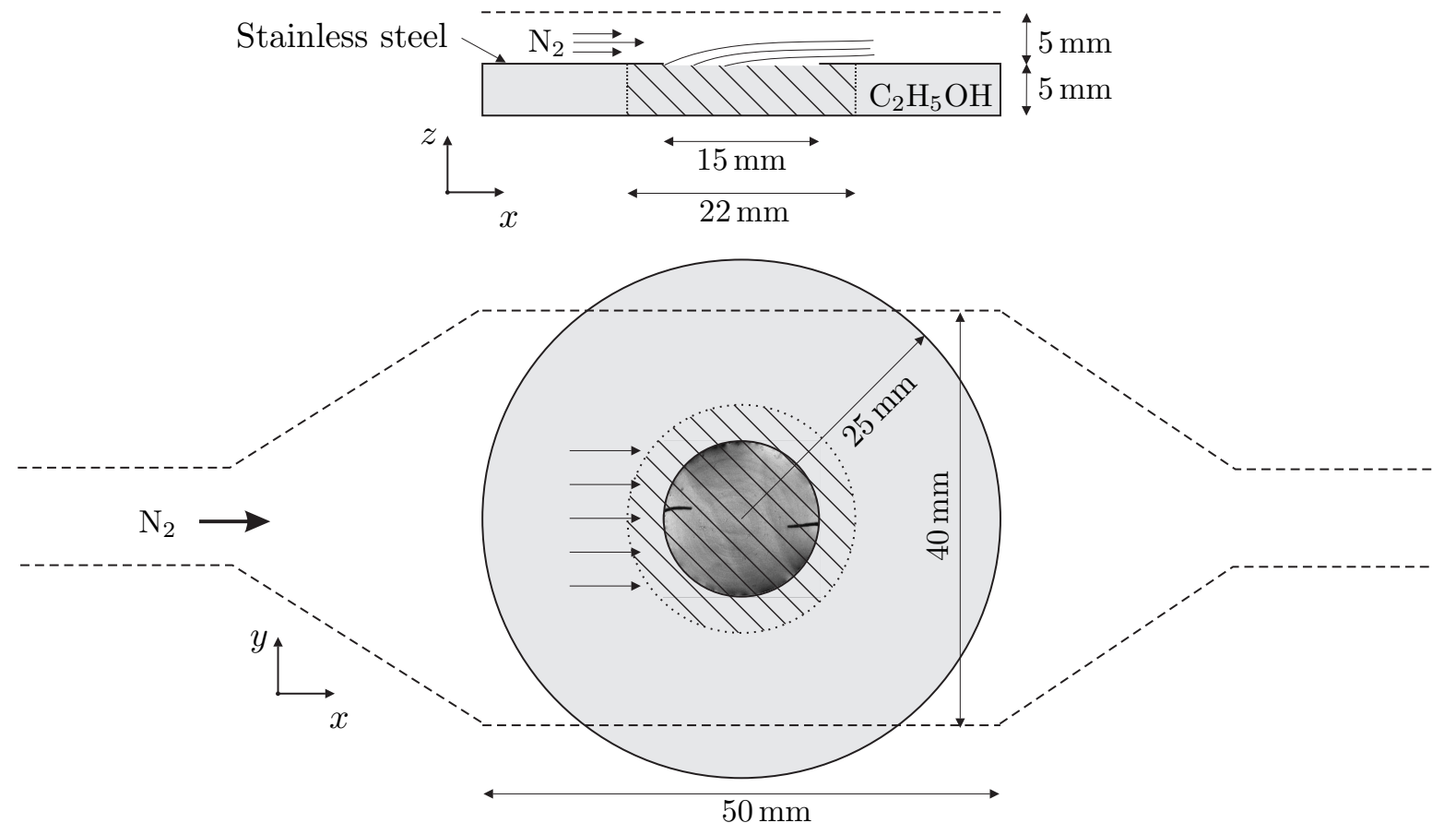

FIG. 1: Side and top views of the experimental cell containing ethyl alcohol (in gray). The hatching indicates the zone in which the three-dimensional temperature field has been reconstructed by tomography. The picture fitting the opening through the stainless steel foil is a Schlieren image and the two black stripes on it are thermocouples mounted in the gas phase. The dashed lines indicate the boundaries of the gas channel above the liquid layer. To fix ideas, the concentration boundary layer of alcohol vapor in the Nitrogen gas phase is represented in the side view.

diameter opening made in a $0.2 \mathrm{~mm}$ stainless steel foil, and removed by a nitrogen $\left(\mathrm{N}_{2}\right)$ flow parallel to the interface. The gas channel is shown in dashed lines. The dimensions of 
the gas channel were chosen to ensure that the gas flow is laminar when reaching the liquid opening. This can be verified by calculating the Reynolds number for the gas based on the channel height, denoted $h_{g}$, namely $R e=u_{g} h_{g} / \nu_{g}$, with $\nu_{g}=1.5 \times 10^{-5} \mathrm{~m}^{2} \mathrm{~s}^{-1}$ the kinematic viscosity of Nitrogen at $20^{\circ} \mathrm{C}$. The gas velocity is estimated from the imposed flow rate $F$ $\left(\mathrm{ml}_{n} / \mathrm{min}\right)$, i.e. $u_{g}=\frac{10^{-6}}{60} \mathrm{~F} /\left(w_{g} h_{g}\right) \mathrm{ms}^{-1}$, where $w_{g}=0.04 \mathrm{~m}$ is the channel width. The Reynolds number is therefore $R e \approx 0.03 F$, so with $F \sim 100 \mathrm{ml}_{n} / \mathrm{min}$ as considered in this study, $R e=O(1)$, which indeed ensures that the flow is laminar.

The gas can also be considered as passive in terms of mechanical coupling since the dynamic viscosity of the gas is two orders of magnitude smaller than the one of the liquid. The contribution to the surface velocity of the shear stress exerted by the gas flow is thus two order of magnitude smaller than $u_{g}$, and negligible compared to the contribution due to the Marangoni stress. In fact, this is true provided the gas flow is not too high, typically $F<1000 \mathrm{ml}_{n} / \mathrm{min}$, which is always the case in this study.

Two optical diagnostics were used in the experiments, both sensitive to refractive index variations within the liquid. The first one is a top-view Schlieren system working in double transmission and reflection on a mirror placed at the bottom of the liquid layer. The Schlieren system allows to measure variations of the refractive indices, hence variation of the temperature within the liquid. Notice that although interface deflections do contribute to the Schlieren image (which is used for flatness control [11]), they can be clearly separated from small-scale ripples we are interested in. The second diagnostic is an optical tomograph using six interferometers spaced $30^{\circ}$ apart around the cell (see schematic in [12]). This instrument measures integrated optical path variations along the different directions, the combination of which enables via a dedicated reconstruction algorithm to determine the three-dimensional temperature field in the liquid layer with some accuracy depending on the complexity of the pattern (see details of the technique in [13-15]). In the present experiment, the resolution of the tomograph is $0.001 \mathrm{~K}$, such that one can safely state that the error is at most one order of magnitude larger, i.e. $0.01 \mathrm{~K}$, which represent $0.1 \%$ of the total temperature range. The effective volume in which the three-dimensional temperature field has been reconstructed corresponds to the hatched zone in Fig. 1.

Figure 2 shows the experimental points considered both in microgravity $(0 \mathrm{~g})$ and during ground-based (1g) campaigns. The control parameters are the gas pressure $P$ (mbar) and the gas flow rate $F\left(\mathrm{ml}_{n} / \mathrm{min}\right)$. As mentioned in the introduction, because of the 


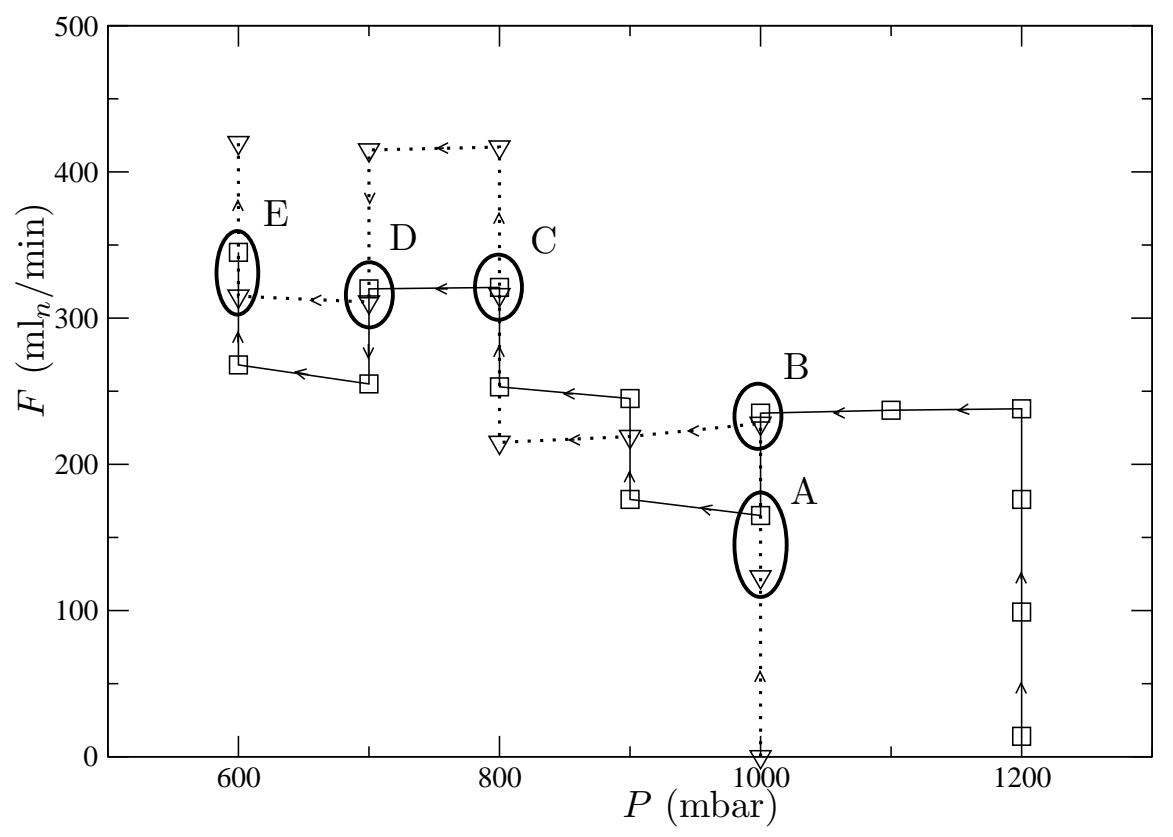

FIG. 2: Experimental scenario in microgravity $(\square)$ and ground-based $(\nabla)$ in the gas pressure/flowrate parameter space. Letters refer to the different results presented in this paper.

short microgravity time of MASER (6 minutes), only transient regimes could be observed. The experimental points were scanned from small to large evaporation rates, i.e. from low- $F /$ large- $P$ to large- $F /$ low- $P$. The ratio $F / P$ thus represents a rough measure of the 'evaporation strength' and will be used as such hereafter. The sampling time assigned at each experimental point was about $15 \mathrm{~s}$ and the transitions represented by solid lines for $0 \mathrm{~g}$ (resp. dotted lines for $1 \mathrm{~g}$ ) in Fig. 2 was about a few seconds.

The experimental set-up incorporated a thermal system consisting of a loop used for controlling the initial temperature in the injection unit and the experiment cell. The temperature was then stabilized at the start of each experiment and measured (through embedded thermocouples) to be $24.8{ }^{\circ} \mathrm{C}$ for the $0 \mathrm{~g}$-experiment and $25.0{ }^{\circ} \mathrm{C}$ for the $1 \mathrm{~g}$-experiment. For the $0 \mathrm{~g}$ experiment, the thermalization system was disconnected at launch and the maximum deviation of the temperature during the whole experiment was not larger than $0.2{ }^{\circ} \mathrm{C}$, indicating a good thermal insulation of the experimental cell with the surrounding.

As mentioned above, the free surface was kept flat, even in the presence of evaporation, thanks to a flatness control system using the average curvature of the meniscus (measured on the Schlieren images) to discriminate between an overfilled and an under-filled liquid surface. 
A dedicated liquid injection unit was then activated to inject or retract liquid accordingly in order to maintain a flat surface (see also [12]). The best resolution of the surface deflection was $10^{-2}$ degree. This procedure additionally allowed to approximately infer the evaporation rate during the experiments, although reliable instantaneous values were not available for the microgravity experiment due to control-induced small-amplitude oscillations. Notice that this additional noise on the measurement did not prevent the experiment itself to be valuable but only reduced the scientific return (less points in the scenario could be explored).

\section{EXPERIMENTAL OBSERVATIONS}

We give below the experimental observations as they were obtained from low to high evaporation rates. The experimental images shown below are snapshots of movies recorded at a frame rate of $24 \mathrm{~Hz}$, which was large enough to capture the interfacial ripple dynamics and exclude stroboscopic effects.

\section{A. Low evaporation rates}

Figure 3 shows Schlieren pictures at low evaporation rate (corresponding to point A and $\mathrm{B}$ in Fig. 2), with the occurrence of thermal ripples. At such low evaporation rates, the ripples are barely visible on snapshots (though they are easily seen by eye in movies). The images have thus been post-processed to determine the position of the ripples. Figure $3 d$ illustrates this post-processing. It consists in removing the background and increasing the contrast, which enables to precisely locate the ripples.

In microgravity, ripples appear to move parallel to the gas flow either downstream, or upstream as the one shown in Fig. $3 a$ (dotted line), but not specifically in alternation. They appear one by one at a rate of about $0.5 \mathrm{~Hz}$ and always from the same site located at the tip of the steady $\mathrm{V}$-shape (solid line), reminiscent to the presence of a large scale convection cell (see also Fig. 4). The origin of these ripples could therefore be explained on the basis of secondary instability of this large-scale convection cell.

The large-scale structure originates from the Marangoni instability triggered by the cooling of the interface by evaporation, which induces a temperature difference $\Delta T$ across the layer. A typical Marangoni number can then be estimated, namely $M a=|\mathrm{d} \sigma / \mathrm{d} T| \Delta T d /(\mu \chi)$, 


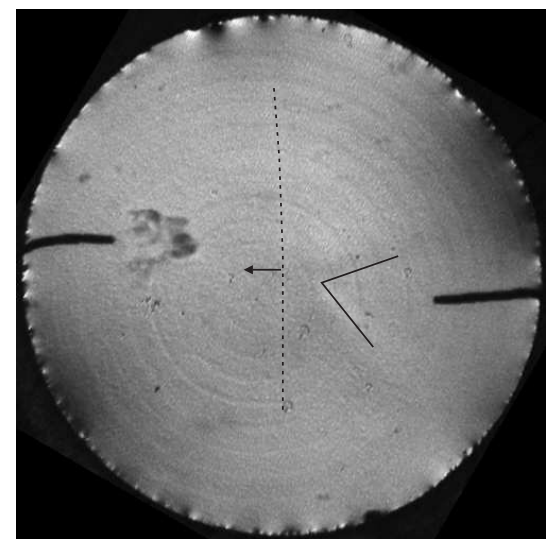

(a) $0 \mathrm{~g}-F=162 \mathrm{ml}_{n} / \mathrm{min}$

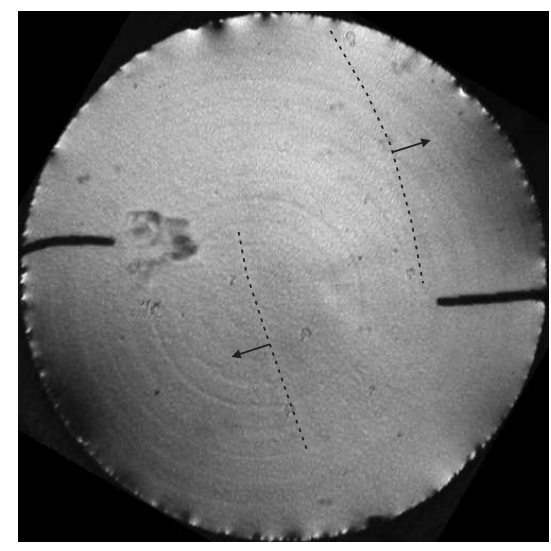

(c) $0 \mathrm{~g}-F=235 \mathrm{ml}_{n} / \mathrm{min}$

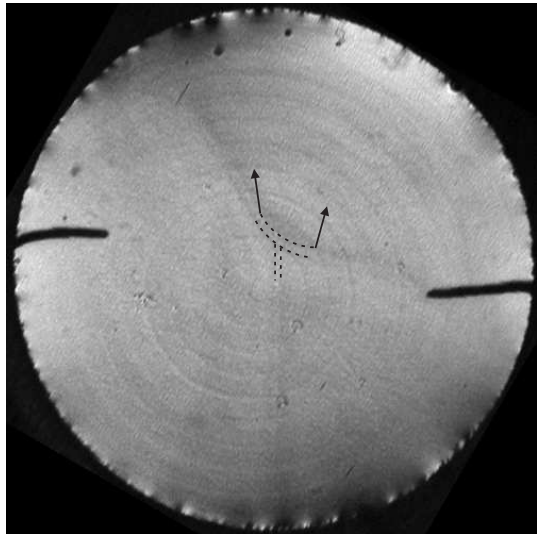

(b) $1 \mathrm{~g}-F=123 \mathrm{ml}_{n} / \mathrm{min}$

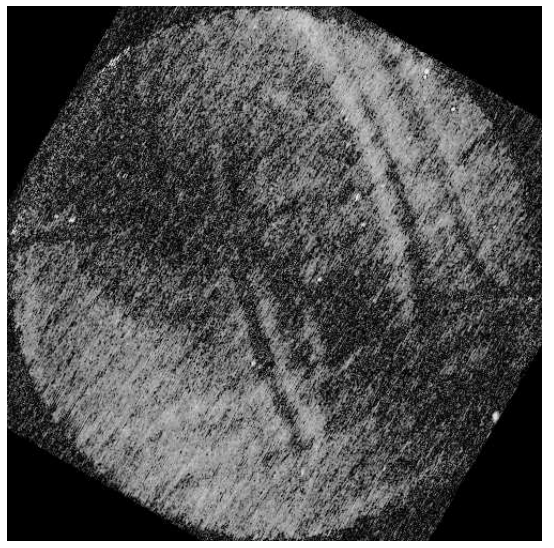

(d) $0 \mathrm{~g}-F=235 \mathrm{ml}_{n} / \mathrm{min}$

FIG. 3: Schlieren pictures obtained for $P=1000$ mbar (Point A and B in Fig. 2). The gas flow $(F)$ is from left to right on each picture. Dashed lines guide the eyes for the position of thermal ripples and the arrows indicate the direction of their motion. The ripple velocities are (a,c,d) $2 \mathrm{~mm} / \mathrm{s}$ and (b) $0.5 \mathrm{~mm} / \mathrm{s}$. In (a) the solid line shows a steady V-shaped structure. Notice in (b) that the thermal ripples are double. The image in (d) is the same as in (c) after post-processing (see text for details).

where $\sigma$ is the surface tension, $d$ is the thickness of the liquid layer, $\mu$ is the dynamic viscosity and $\chi$ is the thermal diffusivity. For ethanol, $|\mathrm{d} \sigma / \mathrm{d} T|=8.8 \times 10^{-5} \mathrm{Nm}^{-1}, \mu=1.2 \times 10^{-3} \mathrm{Pas}$, and $\chi=8.7 \times 10^{-8} \mathrm{~m}^{2} \mathrm{~s}^{-1}$, such that for a typical temperature difference of $\Delta T=0.5 \mathrm{~K}$, the Marangoni number is about 2000, which is much higher than its critical value for the Marangoni-Bénard instability, namely $M a_{c}=O\left(10^{2}\right)$ (see e.g. [3, 9]). Being far above the instability threshold, strongly nonlinear behavior can be expected, such as the devel- 
opment of thermal boundary layers close to the interface [9]. This is why we believe these ripples originate from a secondary instability as said above (and as studied in [9] from a phenomenological model). Nevertheless, we have not yet been able to identify a threshold for these ripples to occur, given the transient nature of our experimental results.

The Schlieren picture for the corresponding ground experiment (Fig. $3 b$ ) shows a $\lambda$-shape structure moving, on the contrary, perpendicular to the gas flow direction. Double ripples — i.e. two Schlieren intensity extrema moving jointly and simultaneously — were generally present at low evaporation rate and disappeared systematically by increasing it. Notice they were also occasionally observed in microgravity (not shown here). Figure 4 shows the reconstructed three-dimensional temperature field obtained by tomography, at the same times that the snapshots of Fig. $3 a-b$. The bottom maps show the temperature field on a horizontal slice located approximately $200 \mu \mathrm{m}$ underneath the free surface.

In $0 \mathrm{~g}$, we observe in Fig. $4 a$ one warm area near the middle of the cell which is deemed to correspond to the center of a single convection cell, while the edge of the opening is cold, though colder at the entrance side of the nitrogen flow, where the concentration boundary layer in the gas is still under development. Indeed, thinner is the concentration boundary layer, larger is the evaporation rate and thus colder is the interface (i.e. the interface temperature at the left side of the picture is lower than that at the right side). Consequently, the interfacial flow is driven by thermocapillary stresses from warmer regions (red regions on Fig. 4, at the center of the opening) to colder regions (green/blue regions at the edge of the opening), sustaining the presence of a large-scale convection cell.

Figure $5 a$ shows typical trajectories of dust particles observed during the $0 \mathrm{~g}$ experiment with the Schlieren camera. According to the temperature distribution measured in a slice close to the interface (Fig. $5 a$ ), particles traveling away from the center are at or near the interface where the flow is dominated by thermocapillarity, i.e. going from hot to cold locations. Particles traveling toward the center are thus somewhere in the bulk, i.e. in the return flow of a large-scale "convective cell". Assuming the particles to follow the local flow, the liquid reaches the center of the cell with a velocity of about $1 \mathrm{~mm} / \mathrm{s}$ and returns away along the interface with a higher velocity of about $3-5 \mathrm{~mm} / \mathrm{s}$. Figure $5 b$ represents the calculated velocity vector field in the $200 \mu \mathrm{m}$ slice taken as if it was the non-deformable interface of an incompressible Stokes flow with tangential stress driven by pure Marangoni effect on it and no-slip conditions on the side and bottom boundaries, as well as on the 

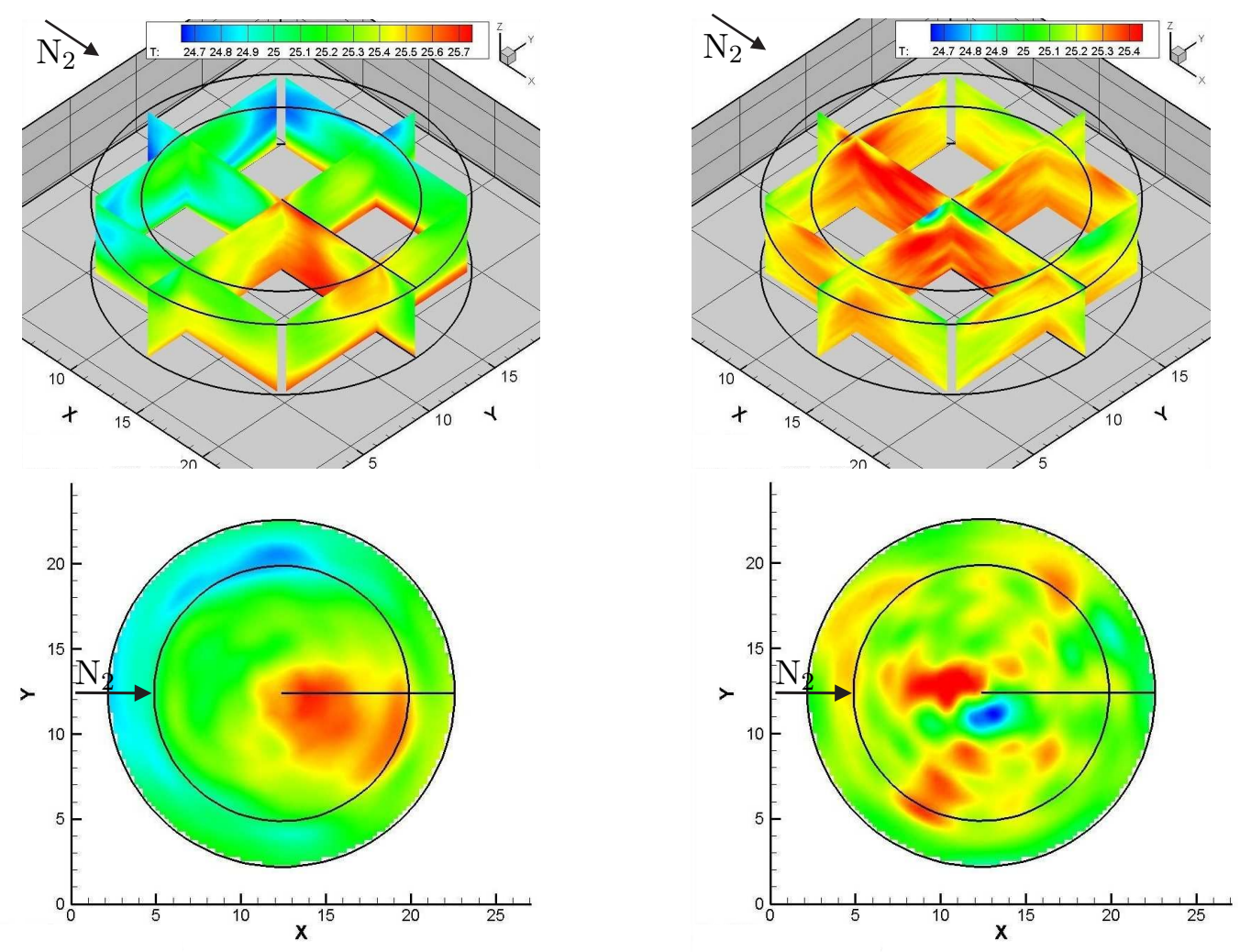

(a) $0 \mathrm{~g}-F=162 \mathrm{ml}_{n} / \mathrm{min}$

(b) $1 \mathrm{~g}-F=123 \mathrm{ml}_{n} / \mathrm{min}$

FIG. 4: Temperature field as reconstructed by tomography for $P=1000 \mathrm{mbar}$. The bottom maps show the upper slice of the three-dimensional temperature field, i.e. at $200 \mu \mathrm{m}$ underneath the interface. The arrows show the gas flow direction.

outside annulus region of the upper boundary (outside the opening). Notice that the Stokes flow assumption applies rigorously in the limit of an infinite Prandtl number $(\operatorname{Pr}=\infty)$ [16], which is certainly not the case here $\left(\operatorname{Pr}=12\right.$ for pure ethyl alcohol at $\left.25^{\circ} \mathrm{C}\right)$, still, it represents a convenient assumption to linearize the system of equations and get some insights of the unknown velocity field from the known surface temperature one. Results show that the liquid flows outwards the hottest spot (in red) in all directions with a velocity that can be as large as $15 \mathrm{~mm} / \mathrm{s}$ (see the reference arrow at the top right corner of Fig. $5 b$ ). If we now assume the ripples to be transported by the interfacial flow, it can explain why they can move upstream and downstream (see for instance Fig. $3 c$ ). 


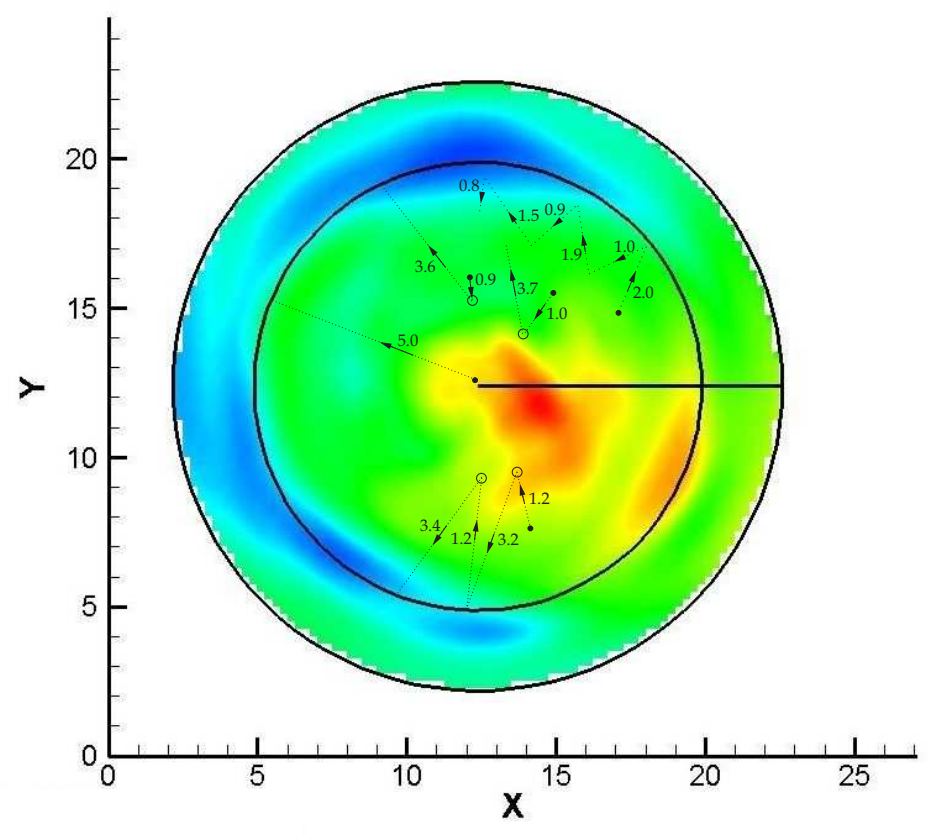

(a)

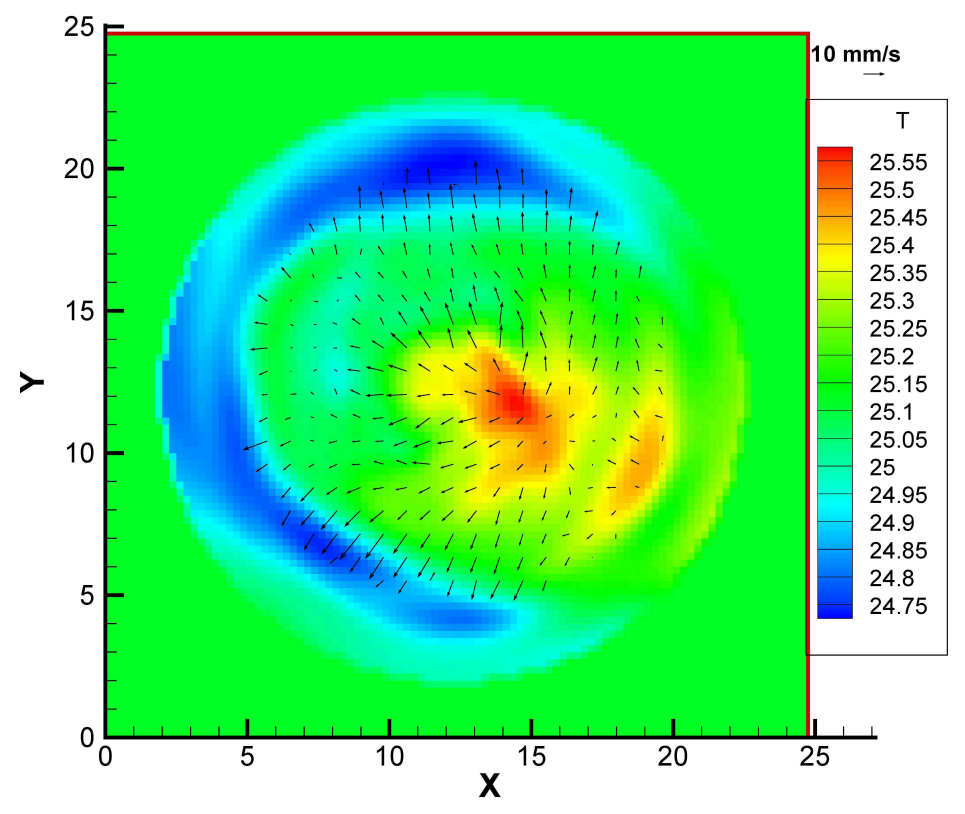

(b)

FIG. 5: Temperature distribution in a slice $200 \mu \mathrm{m}$ underneath the interface for $P=1200 \mathrm{mbar}$ and $F=238 \mathrm{ml}_{n} / \mathrm{min}$ in $0 \mathrm{~g}$. (a) Experimental trajectories of particles in dotted lines with the values of velocities in $\mathrm{mm} / \mathrm{s}$. The arrows pointing inwards are in the bulk and those pointing outwards are at or near the interface. The circles indicate where the particles move from the bulk to the interface along the $z$-coordinate (during a time of about $0.5 \mathrm{~s}$ ). (b) Near-surface velocity field calculated from the measured temperature distribution (see text for details). 


\section{B. Transition to chaotic regimes}

In $1 \mathrm{~g}$, inhomogeneities of the temperature field are much more pronounced than in $0 \mathrm{~g}$ (Fig. 4b). This fact is attributed to buoyancy, which reinforces the flows, in agreement with the fact that the ripples are generally more numerous and more disordered than in $0 \mathrm{~g}$ at identical control parameter values. Care should be taken here however as due to the limited number of views, tomography might not be able to reconstruct more complex patterns arising in $1 \mathrm{~g}$ with sufficient accuracy. Nevertheless, comparison between $0 \mathrm{~g}$ and $1 \mathrm{~g}$ allows to expect that microgravity delays and makes more gradual the onset of "interfacial turbulence", by suppressing buoyancy-driven flow and thus leading to a more "smooth" transition to chaotic regimes, driven then exclusively by thermocapillary effects.

In $1 \mathrm{~g}$ condition, one can assess the importance of buoyancy in the large-scale structure development by calculating the Rayleigh number, $R a=g \alpha \Delta T d^{3} /(\nu \chi)$, where $g$ is the gravitational acceleration, $\alpha$ is the volumetric thermal expansion coefficient and $\nu$ is the kinematic viscosity. For ethanol, $\alpha=7.5 \times 10^{-4} \mathrm{~K}^{-1}$ and $\nu=1.5 \times 10^{-6} \mathrm{~m}^{2} \mathrm{~s}^{-1}$, which leads to $R a \approx 3500$ for a temperature difference of $\Delta T=0.5 \mathrm{~K}$. Since the critical Rayleigh number in our problem can be estimated by considering that our upper surface is a slip-free and insulating boundary, which gives $R a_{c}=669$ (see e.g. $[9,17]$ ), and thus $R a \gg R a_{c}$, there is no doubt buoyancy has a strong influence on the pattern development observed in ground experiments.

The transition to turbulence has been qualitatively defined here as the transition from nearly periodic to disordered (or chaotic) behavior in the observed evolution of thermal ripples at the interface. As mentioned above, no such transition has been found in $1 \mathrm{~g}$ where disordered behavior was observed already for the lowest flow rate $\left(F=100 \mathrm{ml}_{n} / \mathrm{min}\right)$ and at any pressure. In $0 \mathrm{~g}$, such a transition has been identified at point $\mathrm{C}$ in Fig. 2 (in the accuracy limit of the experiment and in a qualitative sense only since each step of the experiment is inherently transient). For lower evaporation rates, ripples occur one by one with an apparent periodicity while moving at similar speed. For higher evaporation rates, the periodicity is broken, the number of ripples varies in time, as well as their speed and motion direction. Figure 6 shows such a behavior in detail. At time $0 \mathrm{~s}$, two ripples, denoted primary ripples hereafter, are present and move parallel to the flow but in opposite directions as indicated by the arrows. At time $0.5 \mathrm{~s}$, a secondary ripple appears perpendicularly and starts interacting 

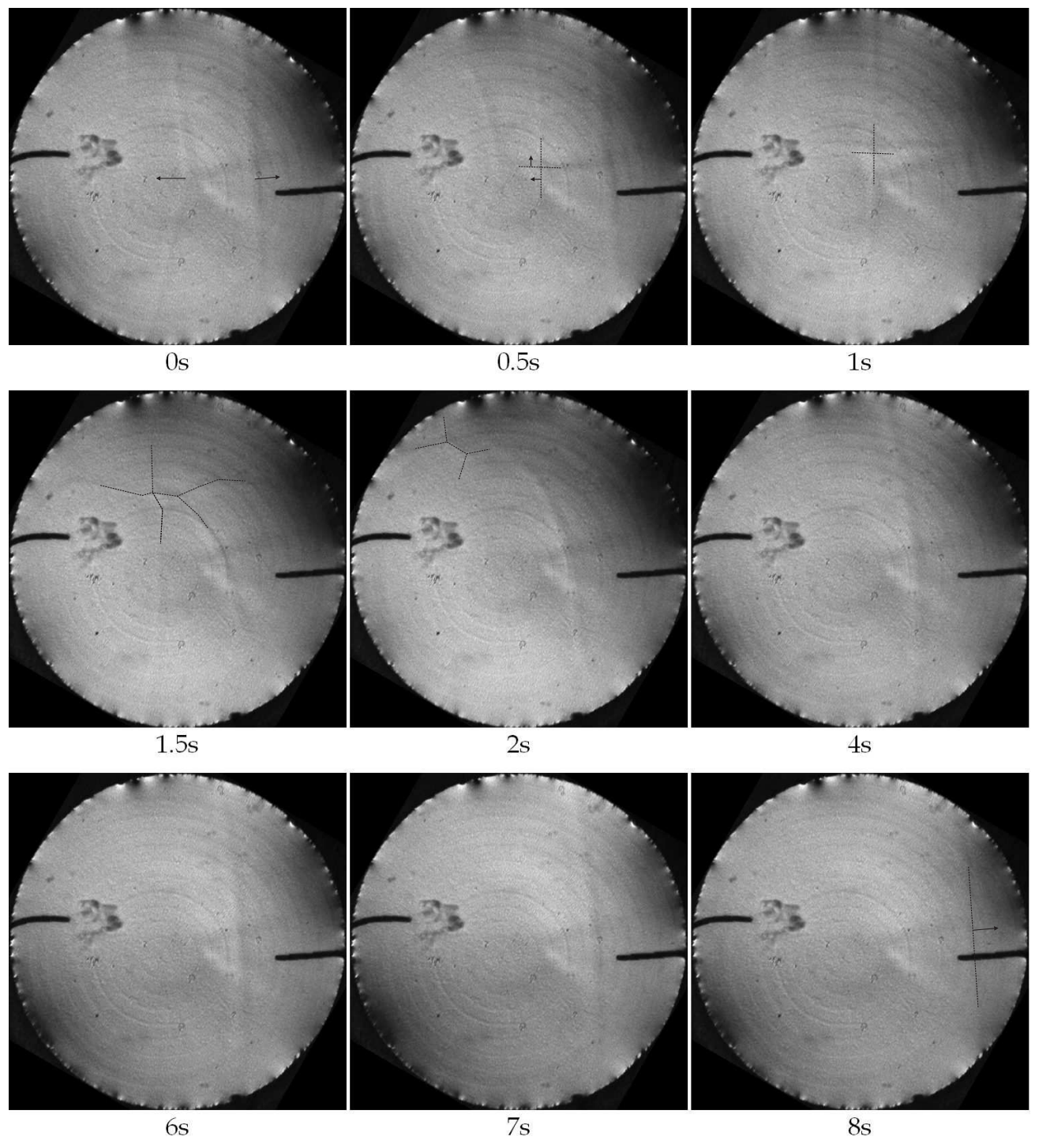

FIG. 6: Schlieren snapshots in $0 \mathrm{~g}, P=800 \mathrm{mbar}$ and $F=319 \mathrm{ml}_{n} / \mathrm{min}$ (point $\mathrm{C}$ in Fig. 2). Gas flow is from left to right on each snapshot. Time is indicated in seconds from the first picture taken as reference. Thin black lines guide the eye for the position of thermal ripples when they are barely visible.

with a new primary ripple that is just born. The intersection is emphasized by the black cross, which first moves upwards due to the motion of the secondary ripple $(1 \mathrm{~s})$, then acquires an upstream (i.e. opposite to the gas flow direction) component when the primary ripple starts to move $(1.5 \mathrm{~s})$, and ends up in a distorted H-shape $(2 \mathrm{~s})$. Based on the calculated velocity field, one observes that the ripple intersection moves with a speed equivalent to the 


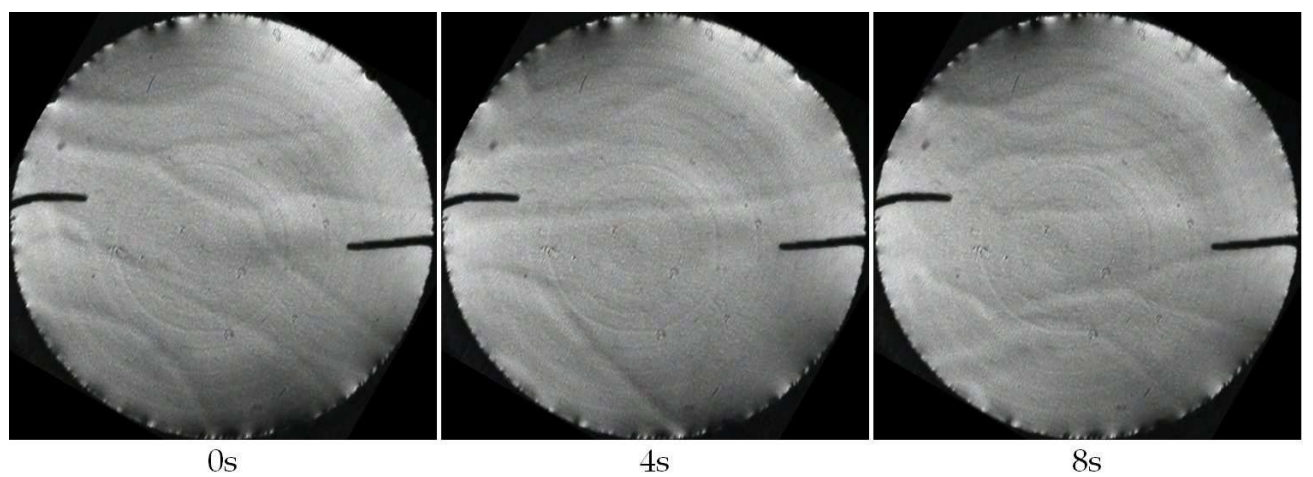

FIG. 7: Schlieren snapshots for $1 \mathrm{~g}, P=700 \mathrm{mbar}$ and $F=316 \mathrm{ml}_{n} / \mathrm{min}$ (point D in Fig. 2). Gas flow is from left to right on each snapshot.

surface velocity.

\section{Intermittent behavior}

Intermittent behavior refers here to stopping down and suddenly restarting of chaotic behavior at intervals. Figure 6 shows such intermittent behavior where chaotic behavior stops for more than $3 \mathrm{~s}$ when a primary ripple stays at rest (from $4 \mathrm{~s}$ to $6 \mathrm{~s}$ ) before moving slowly downstream (from $7 \mathrm{~s}$ to $8 \mathrm{~s}$ ) and giving rise to more disordered bursts afterwards (not shown). Though less obvious, intermittent behavior has also been observed in $1 \mathrm{~g}$ as shown in Fig. 7. Indeed, the chaotic behavior does not disappear totally like in $0 \mathrm{~g}$ but was diminished for some seconds during the time a primary ripple stays at rest (compare the snapshot at $4 \mathrm{~s}$ with those before and after).

\section{High evaporation rates}

At higher evaporation rates, ripples interaction becomes more intense as shown in Fig. 8 for $0 \mathrm{~g}$. We also observe here a transition from a cross to a distorted H-shape (see dotted lines).

Now, in $1 \mathrm{~g}$ and for similar values of the control parameters, the numbers of ripples is much larger and their average frequency of appearance much higher. Figure 9 shows that they even form polygonal patterns at high evaporation rates. Interestingly, most of the 

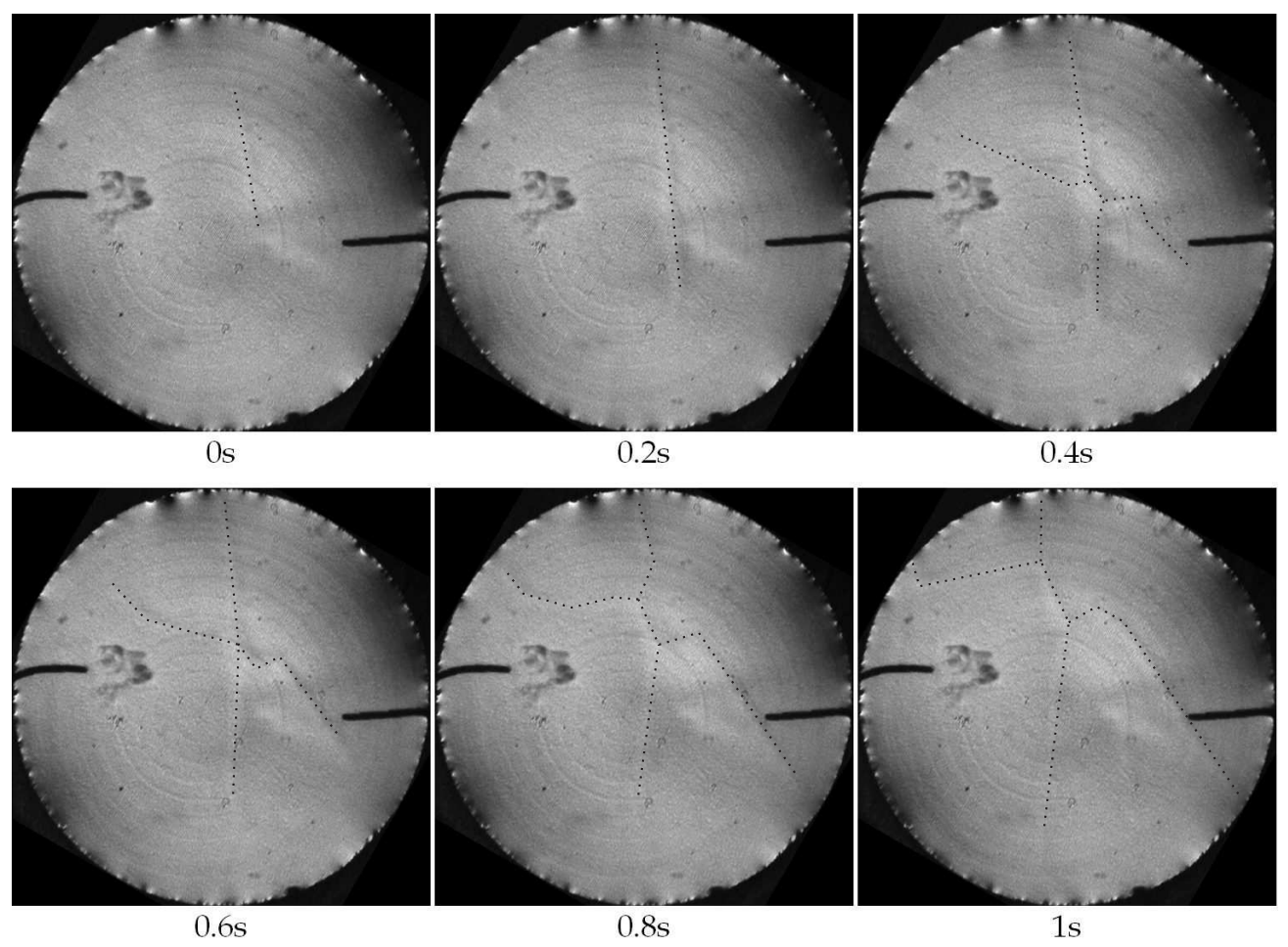

FIG. 8: Schlieren snapshots for $0 \mathrm{~g}, P=600 \mathrm{mbar}$ and $F=345 \mathrm{ml}_{n} / \mathrm{min}$ (point E in Fig. 2). Dotted lines are superimposed on the ripples to guide the eye. Gas flow is from left to right on each snapshot.

ripples are aligned with the gas flow direction in $1 \mathrm{~g}$ while they are perpendicular to it in $0 \mathrm{~g}$. This qualitative difference can be related to the strongly different large-scale thermal structures in $0 \mathrm{~g}$ and $1 \mathrm{~g}$ (compare Fig. $4 a$ and $b$ ) - remember the ripples arise from a secondary instability of these structures. Yet, no explanation has been found to rationalize the different orientation of the primary ripples for $0 \mathrm{~g}$ and $1 \mathrm{~g}$ conditions.

\section{DATA ANALYSIS}

Reconstruction of three-dimensional temperature field has been achieved for all experimental points. Although temperature gradients get stronger at the interface when the evaporation rate increases, no qualitative change has been observed as compared to Fig. 4. Especially in $0 \mathrm{~g}$, the single convection cell seen in Fig. $4 a$ was present during the whole experiment. This confirms that interfacial turbulence due to higher evaporation rates does 


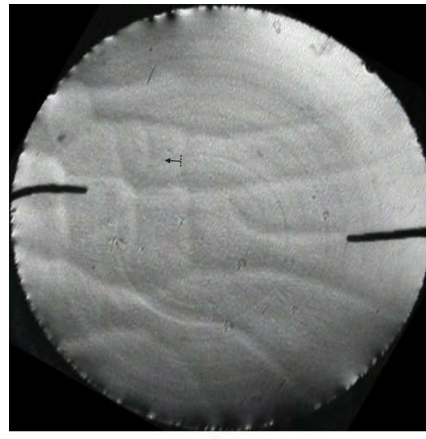

Os

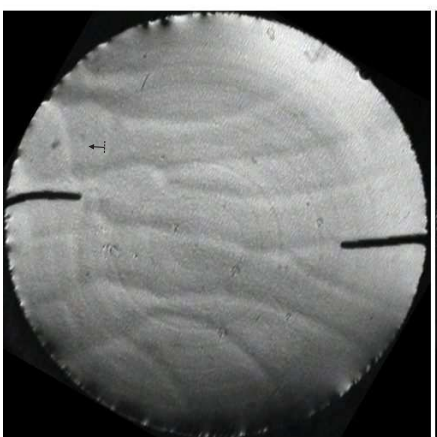

$0.6 \mathrm{~s}$

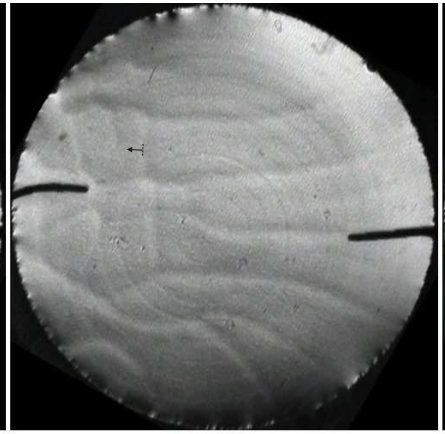

$0.2 \mathrm{~s}$

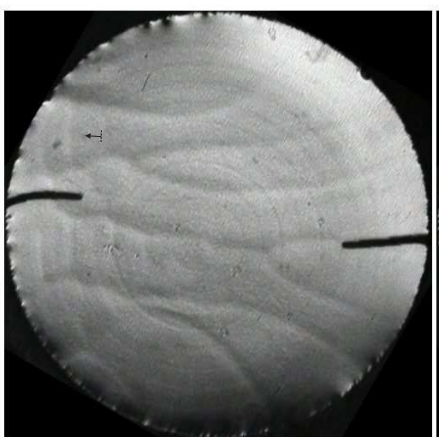

$0.8 \mathrm{~s}$

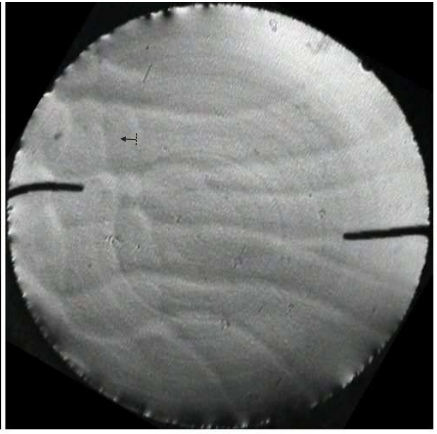

$0.4 \mathrm{~s}$

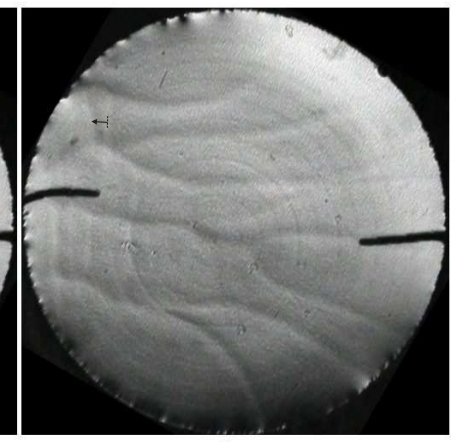

Is

FIG. 9: Schlieren snapshots for $1 \mathrm{~g}, P=700 \mathrm{mbar}$ and $F=316 \mathrm{ml}_{n} / \mathrm{min}$ (point D in Fig. 2). Gas flow is from left to right on each snapshot. The arrows show the displacement of a ripple in the direction opposite to the direction of the gas flow, while most of the ripples are parallel to it.

not significantly affect the large-scale flow in the bulk. The penetration depth of thermal ripples can thus be assessed to be about $200 \mu \mathrm{m}$, which is the limit of spatial resolution of the tomograph in the $z$-direction (yet, they can be detected by eye on the interferograms).

Figure 10 reports for both microgravity and ground experiments the maximum temperature difference recorded on the upper slice of the reconstructed temperature field (i.e. just underneath the interface) for various evaporation rates represented arbitrarily by the ratio $F / P$. It shows that a temperature difference of about $1 \mathrm{~K}$ sets up even for small evaporation rates and that the increase is roughly linear in both $1 \mathrm{~g}$ and $0 \mathrm{~g}$. The fact that the slope is less pronounced in $1 \mathrm{~g}$ than in $0 \mathrm{~g}$ (by a factor three) might be due to the additional mixing induced by the buoyancy effect. For comparison purpose, we also report in Fig. 10 the data points corresponding to simulations performed in the next section. 


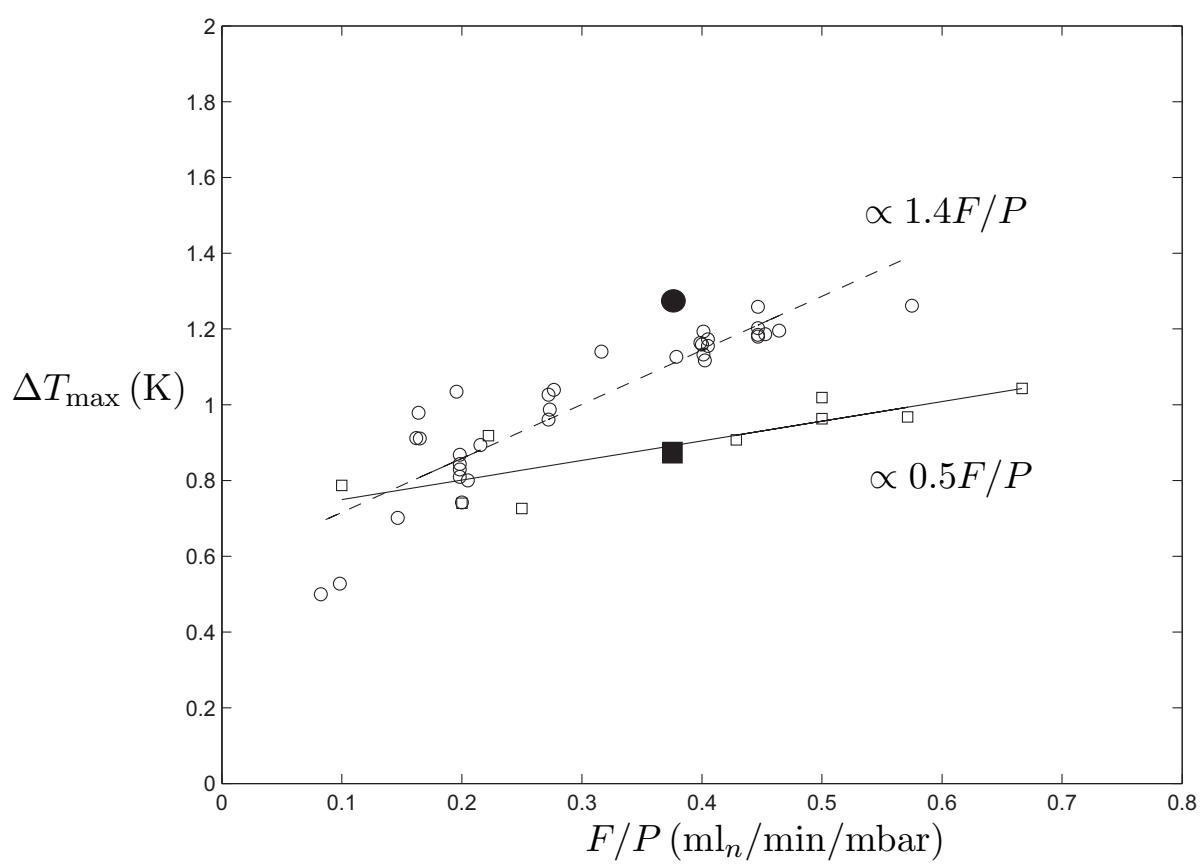

FIG. 10: Maximum temperature difference $\Delta T_{\max }$ at the upper slice versus the 'evaporation strength' $F / P$. Solid (resp. dashed) lines are linear interpolations of the experimental values represented by squares (resp. circles), obtained by tomography in $1 \mathrm{~g}$ (resp. 0g). Filled symbols are obtained from numerical simulations presented in section V.

\section{MODEL OVERVIEW AND NUMERICAL RESULTS}

Numerical simulations have been performed using the commercial software FLUENT based on the volume of fluid method and accounting for the dynamics of both the liquid and the gas phases. The three-dimensional geometry for the numerical simulations has the same dimensions and general features as those of the experimental cell sketched in Fig. 1. The numerical model includes the Navier-Stokes and the energy equations in both the liquid and the gas phases, in addition with the vapor concentration equation in the gas phase. The only external field that is considered is the gravitational one, when present. The effect of evaporation is also taken into account at the liquid-gas interface in a way described in [3], though here in three-dimensions.

Boundary conditions include the no-slip condition at the walls of the liquid cell and the gas channel, as well as at both sides of the stainless steel foil. At the liquid-gas interface, the mass conservation condition and the tangential stress balance including thermocapillary 
(Marangoni) stress are imposed. The interface is assumed non-deformable, horizontal, flat and motionless so the normal stress balance can be ignored. A constant flow rate is imposed at the inlet of the gas channel and a free flow condition is prescribed at the outlet.

The temperature is set constant at the bottom of the liquid cell, while the other walls of the liquid cell and of the gas channel are considered adiabatic. Indeed, despite the thermalization during the experiment (that was only effective for the $1 \mathrm{~g}$ condition as explained in section II), the lateral walls of the cell are made in glass and are quite thick such that thermal transfer should be weak, i.e. low thermal conductivity and low heat transfer coefficient on the other side of the walls. This argument also applies for the walls of the gas channel. In consequence, all the external surfaces are well isolated thermally. In any case, the lateral walls being quite far from the zone of interest, the choice of the thermal boundary condition (isothermal vs. adiabatic) does not influence significantly the results. The only wall for which the adiabatic condition cannot be a fair approximation is for the stainless steel foil separating the liquid and the gas, especially near the liquid opening. Nevertheless, the good agreement between numerical and experimental results that we present hereafter let us believe that the consequences of accounting properly for the heat transfer through the stainless steel foil are not significant, at least in the parameter ranges considered in the present study.

At the liquid-gas interface, the balance of heat fluxes (including latent heat consumption) and the continuity of the temperature are imposed. It is also assumed that local equilibrium prevails at the interface, which is impermeable to the nitrogen present in gas phase. In addition with interfacial boundary conditions given in $[3,9]$, more details regarding their implementation in the special geometry treated here can be found in [18].

For the discretization, a total number of 430756 mesh points has been used with a refinement of the mesh close to the boundaries where the gradients of temperature and vapor mass fraction are the strongest. This number of computational points has been chosen according to a mesh sensitivity test ensuring the convergence of the solutions. The average characteristic length of the control volumes is therefore $500 \mu \mathrm{m}$ except in proximity of the interface where the mesh is refined (typical size of $250 \mu \mathrm{m}$ ).

The simulation has been initialized by filling the gas channel with pure nitrogen and the liquid cell with pure ethyl-alcohol, both at rest. The temperature of the whole cell was also set at $25^{\circ} \mathrm{C}$ at initial time. We note that no disturbances have been artificially imposed, 
although the numerical errors (always present due to the truncation of the derivative and flux terms in the numerical discretization) could have some effects in triggering the rippleforming process. The numerical images that are plotted in this section correspond to a time after which the large-scale flow structures are already established (about 40s), even though an evolution occurs on a longer timescale of the order of the thermal diffusion timescale (of the order $250 \mathrm{~s})$.

Simulations presented in this section have been performed for $P=800 \mathrm{mbar}$ and $F=300 \mathrm{ml}_{n} / \mathrm{min}$ (corresponding to point $\mathrm{C}$ in Fig. 2). Figure 11 shows snapshots of the interfacial temperature field obtained for $0 \mathrm{~g}$. Comparing to experimental results given in Fig. 6, we see similarly the presence of vertical primary ripples moving slowly from the place they are generated (see from $4 \mathrm{~s}$ to $8 \mathrm{~s}$ ) and then moving faster while approaching the edges of the opening where surface tension gradients are the largest (see e.g. the primary ripple on the left at $2 \mathrm{~s}$ which has totally disappeared at $4 \mathrm{~s})$. The fact that thermal ripples are found by direct numerical simulations and that their dynamics compare qualitatively well with experimental results is quite remarkable at least as far as the basic mechanisms are concerned, i.e. secondary instability of large-scale convection cell and Marangoni-driven ripple motion along the interface. It also shows that the system of equations and boundary conditions used in the code is a relevant physical model. Consequently, additional information can then be obtained from simulations, as for instance the fact that thermal ripples appear systematically colder than the interface temperature, which could not be assessed experimentally.

Looking now at the velocity field at a given time for the same conditions, numerical results plotted in Fig. 12 compare again qualitatively well with the velocity vectors reconstructed in the infinite-Prandtl-number limit from the measured temperature field (see Fig. 5b). The fact the surface velocity obtained from numerical simulations are consistent with the measured surface velocity confirms that the thermal Marangoni effect is a dominant effect in the dynamics of interfacial ripples. This fact can also be verified by estimating the typical interfacial velocity induced by the Marangoni stress, namely

$$
u_{s} \sim\left|\frac{\mathrm{d} \sigma}{\mathrm{d} T}\right| \frac{\Delta T}{\mu} .
$$

For ethanol and a temperature difference of $\Delta T=0.5 \mathrm{~K}$, one finds $u_{s} \sim 1 \mathrm{~cm} / \mathrm{s}$, which is indeed consistent with both the numerical simulations and the experimental measurements. 


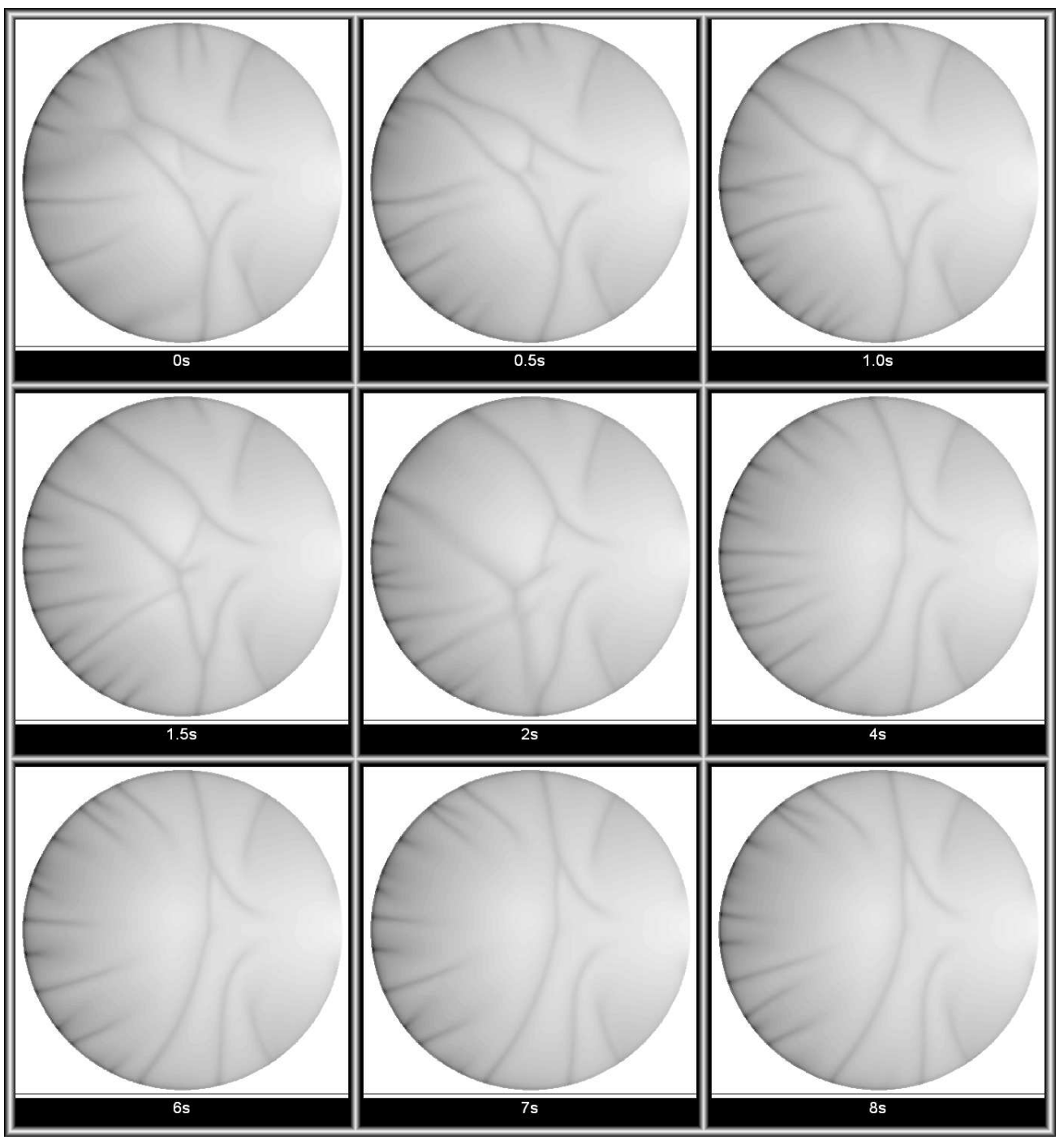

FIG. 11: Evolution of the temperature field at the interface obtained with direct numerical simulations for $P=800 \mathrm{mbar}, F=300 \mathrm{ml}_{n} / \mathrm{min}$ and in $0 \mathrm{~g}$ (Point $\mathrm{C}$ in Fig. 2). Time is indicated in seconds from the first picture taken as reference, i.e. after an initial regime of about $40 \mathrm{~s}$. Dark zones are colder than bright ones with $\Delta T_{\max }=1.3$ as reported in Fig. 10.

Typical pathlines have also been computed from numerical results and shown in Fig. 12 . They are drawn in perspective (out of plane with respect to the interface) to assess the threedimensional trajectory indicating a spiral motion as inferred experimentally from particles motion (see Fig. 5a). The velocity magnitude along the pathlines also turns out to be reminiscent of the experimental observations, i.e. slow and deep in the bulk for those moving toward the center of the cell while fast and near the interface for those moving toward the 

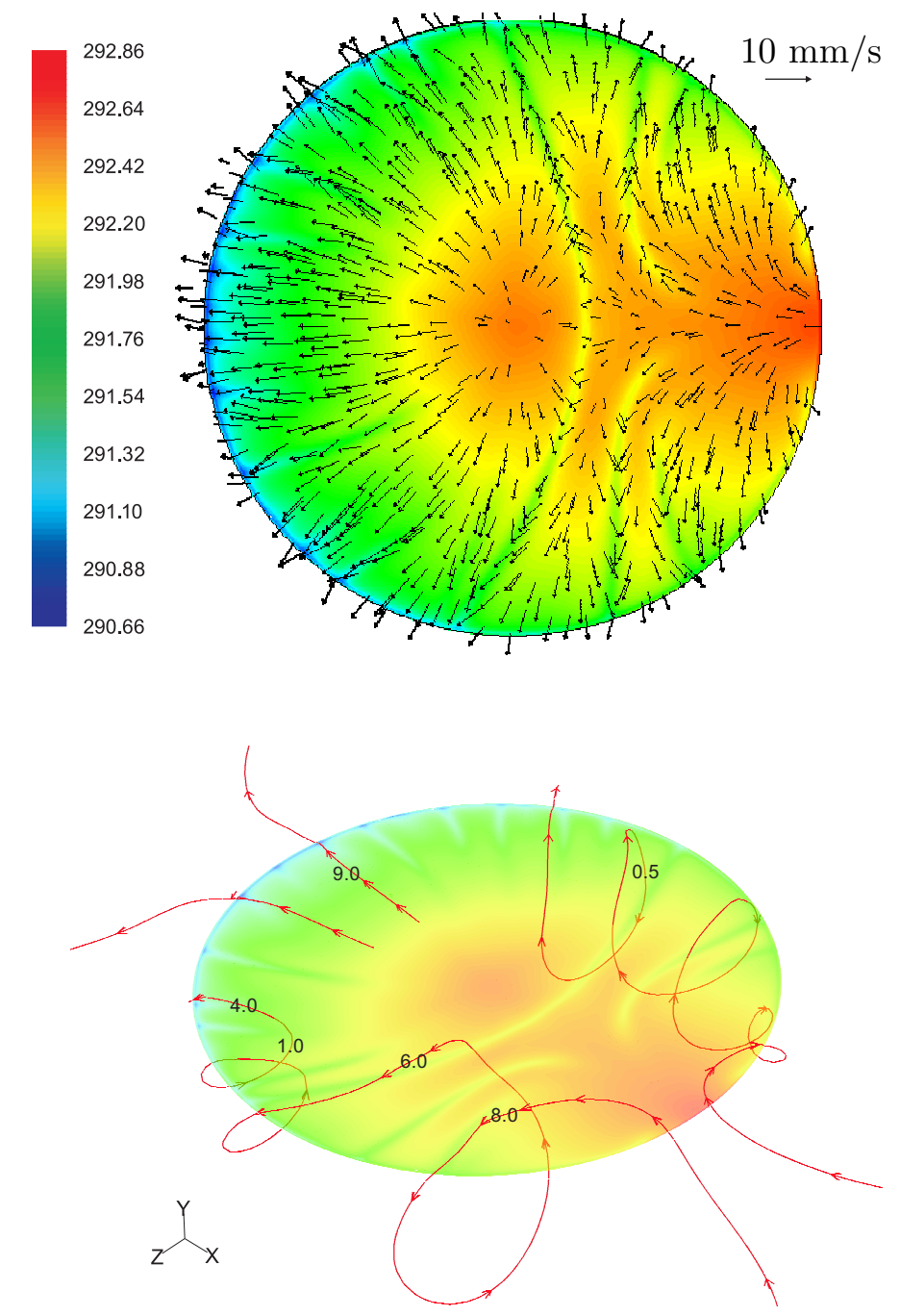

FIG. 12: Temperature and velocity fields at the interface obtained from direct numerical simulations in $0 \mathrm{~g}$ for $P=800 \mathrm{mbar}$ and $F=300 \mathrm{ml}_{n} / \mathrm{min}$ (Point $\mathrm{C}$ in Fig. 2): (top) velocity vectors; (bottom) three-dimensional path lines (i.e. out-of-plane) with typical values of the velocity in $\mathrm{mm} / \mathrm{s}$.

edges of the opening.

Finally for the same conditions as above, we present in Fig. 13 simulation snapshots for $1 \mathrm{~g}$ conditions. It shows vertical ripples moving essentially parallel to the gas flow direction (see from $2 \mathrm{~s}$ to $2.75 \mathrm{~s}$ ) with a larger speed than the horizontal primary ripples that are nearly immobile during the time of the sequence. This numerical result is qualitatively similar to the experimental observations where the primary ripples have a different orientation in $1 \mathrm{~g}$ than in $0 \mathrm{~g}$ conditions. Numerical results in $1 \mathrm{~g}$ also shows more cellular-like polygonal patterns (to be compared with Fig. 9). 


\section{CONCLUSIONS}

In complement to the first observations reported in [11], we have shown in this paper additional experimental evidence of thermal structures in the form of short-scale ripples at a free surface of an evaporating liquid. Microgravity conditions allowed us to scrutinize different regimes by varying the evaporation rate from low to high values, such that the transition to chaotic behavior (or "interfacial turbulence") could be qualitatively identified. Thanks to Schlieren visualization of the interface, three-dimensional tomographic measurements of the temperature field in the bulk and tracking of dust particles, we collected sufficient information to get a good picture of the Marangoni-driven velocity field, which could then be inferred from direct numerical simulations. Details such as the interfacial temperature and velocity fields, spiral-like particle pathlines and ripples motion were found to be in good qualitative agreement. We thus believe that the present work and the consistent comparisons between experimental data and numerical simulations provide a significant progress in the understanding of the mechanisms responsible for the occurrence of interfacial thermal ripples, and the development of interfacial turbulence found for both $0 \mathrm{~g}$ and $1 \mathrm{~g}$ conditions. Because of the short microgravity time of the sounding rocket experiment (6 minutes), only transient regimes could be observed however. Stationary regimes are thus planned to be investigated during the CIMEX-1 experiment on board the International Space Station.

\section{Acknowledgments}

We would like to thank the referees for their valuable suggestions. We would like to thank the whole ESA sounding rocket staff and the whole ITEL 2 team at the Swedish Space Corporation. We are also grateful to Jean Charles Dupin for his help in preparing some figures. We wish to acknowledge the support of E.S.A. (European Space Agency) and of BELSPO (Belgian Federal Science Policy Office) through the CIMEX (Convective Interfacial Mass Exchange) Research Program. B.S. and P.C. acknowledge financial support of the "Fonds de la Recherche Scientifique - FNRS".

[1] J. Berg, A. Acrivos, and M. Boudart, Adv. Chem. Eng. 6, 61 (1966). 
[2] T. Molenkamp, Ph.D. thesis, University of Groningen (1998).

[3] B. Haut and P. Colinet, J. Colloid Interface Sci. 385, 296 (2005).

[4] E. Sultan, A. Boudaoud, and M. Ben Amar, J. Fluid Mech. 543, 183 (2005).

[5] H. Palmer, J. Fluid Mech. 75, 487 (1976).

[6] A. Prosperetti and M. Plesset, J. Fluid Mech. 27, 1590 (1984).

[7] J. Burelbach, S. Bankoff, and S. Davis, J. Fluid Mech. 463-494, 195 (1988).

[8] H. Mancini and D. Maza, Europhys. Lett. 66, 1 (2003).

[9] P. Colinet, J. Legros, and M. Velarde, Nonlinear Dynamics of Surface-Tension-Driven Instabilities (Wiley-VCH, New-York, 2001).

[10] K. Löth, B. Larsson, H. Schneider, O. Janson, Y. Houltz, P. Colinet, C. Iorio, L. Joannes, and O. Dupont, in Proc. of the 53rd International Astronautical Congress, 10-19 October, Houston, Texas (The World Space Congress, 2002).

[11] P. Colinet, L. Joannes, C. Iorio, B. Haut, M. Bestehorn, G. Lebon, and J. Legros, Advances in Space Research 32, 119 (2003).

[12] O. Janson, M. Broxvall, K. Löth, P. Anderson, Y. Houltz, P. Colinet, B. Scheid, L. Joannes, B. Saul, B. Haesendonck, et al., in Proc. of the $1^{\text {th }}$ ESA Symposium on European Rocket \& Balloon Programmes and Related Research, 30 may - 2 june, Sandefjord, Norway (2005), full text can be found on 'http://homepages.ulb.ac.be/ bscheid/Jan05.pdf'.

[13] L. Joannes, O. Dupont, F. Dubois, P. Colinet, and J. Legros, in Proc. of the $9^{\text {th }}$ Int. Symp. on Flow Vizualization (Edinburgh, 2000), p. 428, cD-ROM Proceedings (ISBN 0-9533991-1-7).

[14] L. Joannes, O. Dupont, S. Gorza, P. Colinet, J. Legros, T. Lanen, and H. Oksuzoglu, in Proc. of Fringe 2001, $4^{\text {th }}$ Int. Workshop on Automatic Processing of Fringe Patterns (2001), pp. $506-513$.

[15] P. Colinet, L. Joannes, C. Iorio, O. Kabov, O. Dupont, S.-P. Gorza, and J. Legros, in Proc. of the $15^{\text {th }}$ ESA Symposium on European Rocket \& Balloon Programmes and Related Research (Biarritz, Spain, 2001), vol. ESA SP-471, pp. 423-427.

[16] A. Thess and S. Orszag, J. Fluid Mech. 283, 201 (2005).

[17] D. Nield, J. Fluid Mech. 19, 341 (1964).

[18] C. Iorio, O. Goncharova, and O. Kabov, Microgravity Sci. Technol. 21, S313S319 (2009). 


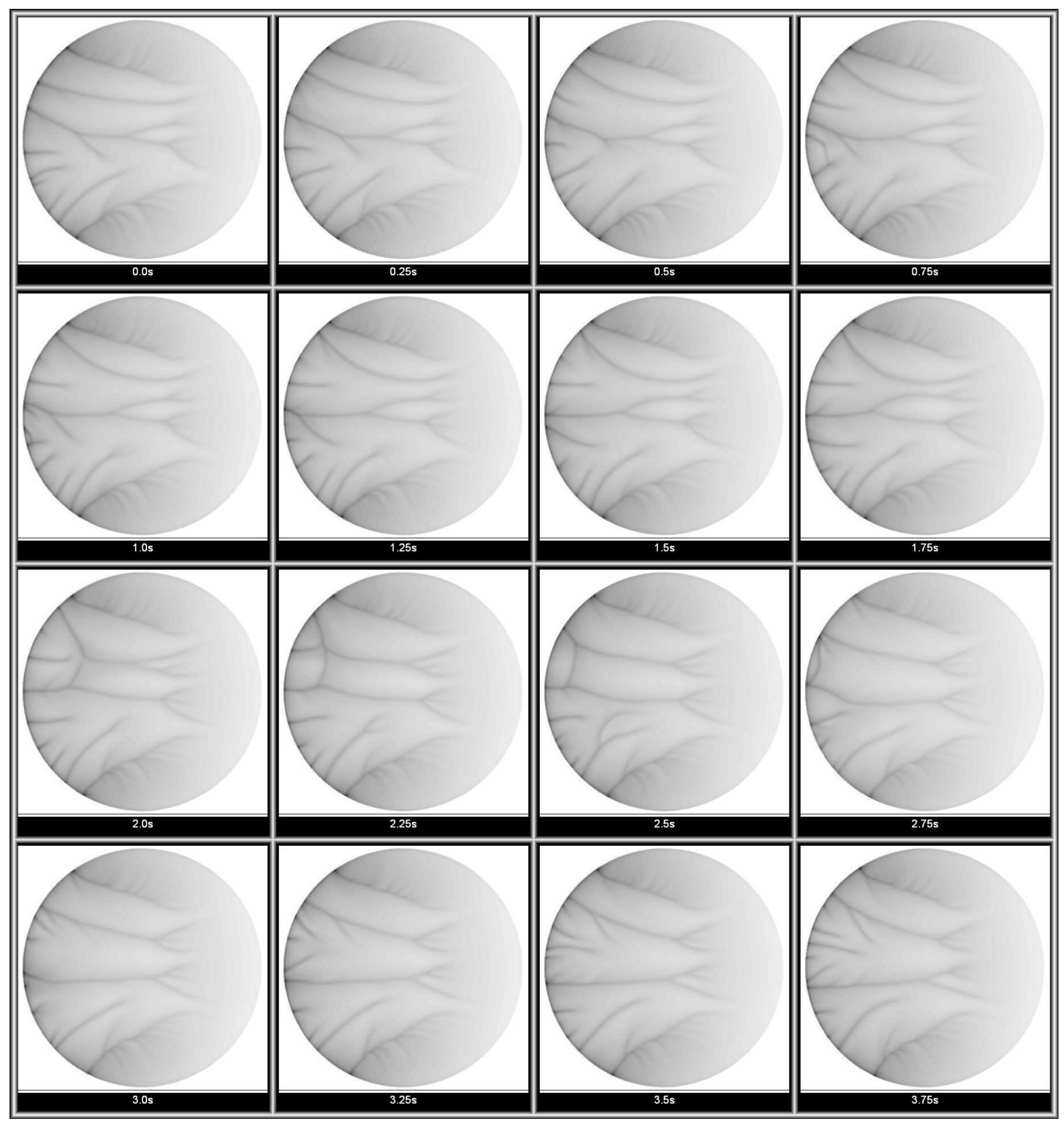

FIG. 13: Temperature field at the interface obtained with direct numerical simulations in $1 \mathrm{~g}$ for $P=800 \mathrm{mbar}$ and $F=300 \mathrm{ml}_{n} / \mathrm{min}$ (Point C in Fig. 2). Time is indicated in seconds from the first picture taken as reference, i.e. after an initial regime of about $40 \mathrm{~s}$. Dark zones are colder than bright ones with $\Delta T_{\max }=0.85$ as reported in Fig. 10 . 